\title{
Ethnic and Age Disparities in Patients Taking Long-acting Injectable Atypical Antipsychotics
}

\author{
Mateen Soleman ${ }^{1}$, Nikki Lam ${ }^{2}$, Benjamin K. Woo ${ }^{3}$ \\ 1. Western, Western University 2. College of Medicine, Northeast Ohio Medical University (NEOMED) 3. UCLA
}

Corresponding author: Benjamin K. Woo, bewoo@dhs.lacounty.gov

\section{Abstract \\ Introduction}

This study will determine whether different ethnicities and different age groups receive equal amounts of long-acting atypical antipsychotics in comparison to their oral equivalents.

\section{Methods}

Secondary analyses of data from the Los Angeles County Department of Health Services Electronic Health Record (total N=63,134 inpatient visits) were performed. Chi-squared statistics were used to compare ethnicity and age with the use of either risperidone injectable or paliperidone palmitate (r-LAIs) versus risperidone oral.

\section{Results}

Among the 63,134 total inpatient visits, there were 3,011 patient visits that included the use of an atypical antipsychotic. Of these 3,011 visits, 452 (15.0\%) were on r-LAIs and 2,559 (85.0\%) were on risperidone oral. No statistically significant disparities were identified with the use of r-LAIs as compared to oral risperidone amongst ethnic groups (chi-square $=0.88, \mathrm{df}=3, \mathrm{p}=0.831$ ). However, there was a statistically significant difference with the use of r-LAIs as compared to oral Risperidone amongst age groups, favoring younger patients (chi-square $=13.46, \mathrm{df}=3, \mathrm{p}<0.004)$.

\section{Conclusion}

Our data indicate a lack of ethnic disparities in prescribing long-acting atypical antipsychotics and an increased percentage of younger patients being treated with atypical depot antipsychotics over their oral equivalents.

Received 09/25/2017

Review began 10/06/2017 Review ended 10/10/2017 Published 10/12/2017

๑) Copyright 2017 Soleman et al. This is an open access article distributed under the terms of the Creative Commons Attribution License CC-BY 3.0., which permits unrestricted use, distribution, and reproduction in any medium, provided the original author and source are credited.
Categories: Psychiatry, Quality Improvement

Keywords: long-acting injections, antipsychotics, racial disparities, duration of untreated psychosis, asian americans, schizophrenia, inpatient psychiatry, psychopharmacology

\section{Introduction}

Long-acting injectable antipsychotic medications (LAIs) have been proven to be both safe and effective for patients suffering from schizophrenia [1]. LAIs carry additional benefit in ensuring medication delivery among patients with surreptitious non-adherence. In patients with schizophrenia, non-adherence rates are estimated to be in the range of 40-50\% [2]. Clinical Antipsychotic Trials of Intervention Effectiveness (CATIE) revealed a 74\% rate of discontinuation of oral antipsychotics within six months of use, necessitating the role of depot antipsychotics [3]. LAIs also relieve the burden of taking daily medications, prevent complete loss of effectiveness for missed doses, and enable clinicians to track noncompliance [3-4].

Various reports have revealed racial disparities amongst those receiving LAIs [5-7]. For instance, individuals from ethnic minority groups were less likely than Caucasians to receive atypical LAIs for schizophrenia or other debilitating psychiatric illness [5]. A meta-analysis demonstrated that although the overall use of LAIs was comparable across ethnic groups, Latino and black patients were less likely than non-ethnic minorities to be treated with second-generation LAIs [7]. Eliminating any racial or ethnic disparities should remain a national goal for prescribing clinicians.

Many patients treated during their first episode of schizophrenia respond favorably to antipsychotics and can achieve appreciable levels of symptom remission within the first year [8]. The time period during which patients manifest psychotic symptoms prior to treatment, known as the duration of untreated psychosis (DUP), has been found to be associated with poor clinical outcomes [9]. Rapid implementation of LAIs has been shown to lessen the severity and duration of acute psychotic exacerbations [10]. Unfortunately, LAIs are generally underutilized, raising concern about whether target age demographics are being adequately addressed [11]. 
This study aims to compare risperidone injectable and paliperidone Palmitate (two atypical long-acting injectable antipsychotics), with risperidone oral to determine whether there lies a disparity with prescribing atypical LAIs within ethnic or age groups amongst a county inpatient population. Furthermore, since atypical LAIs have been shown to be efficacious for first-episode psychosis, we sought to examine whether the highest-risk age range (18 - 34 years old) was being targeted with these long-acting formulations.

\section{Materials And Methods}

The study sample consists of all patients aged 18-64 years receiving either risperidone injectable or paliperidone palmitate (r-LAIs), and risperidone oral across four years (2013 - 2017) within the Los Angeles County Department of Health Services (LAC DHS) electronic health record from a combined pool of 63,134 total inpatient visits. The LAC DHS is a regional, integrated system of providers, clinics, and hospitals that delivers quality, community-based care. The basic sampling unit is the physician-patient encounter or visit. This research used pre-existing, de-identified data and was deemed exempt research involving human subjects by the LAC DHS.

Data collected over a four-year period included patient ethnicity, age range, and use of r-LAIs and risperidone oral. Individuals were sub-classified into the following categories: age (18-34, 35-44, 45-54, and 55-64 years) and ethnicity (Asian, black, white, and other). This data was analyzed using chi-squared testing to compare ethnicity and age with the use of $\mathrm{r}$ - LAIs versus risperidone oral. Data were entered and analyzed using IBM SPSS Statistics v. 21.0 (Armonk, NY).

\section{Results}

Among the 63,134 total inpatient visits, there were 3,011 patient visits that included the use of an atypical antipsychotic. Of these 3,011 visits, 452 (15.0\%) were on r-LAIs. Of this group, 281 (62.2\%) were male and 171 (37.8\%) were female. Of the 3,011 visits, 2,559 (85.0\%) were on risperidone oral. Of this group, 1,683 (65.8\%) were male and 876 (34.2\%) were female.

Table 1 summarizes the use of r-LAIs or risperidone oral amongst different ethnicities and ages. No statistically significant disparities were identified with the use of r-LAIs as compared to oral risperidone amongst ethnic groups (chi-square $=0.88, \mathrm{df}=3, \mathrm{p}=0.831$ ), however, there was a statistically significant difference with the use of r-LAIs as compared to oral risperidone amongst age groups, favoring younger patients (chi-square $=13.46, \mathrm{df}=3, \mathrm{p}<0.004$ ).

\begin{tabular}{|c|c|c|c|c|c|c|c|}
\hline & \multicolumn{2}{|c|}{ Paliperidone palpitate or injectable risperdone $(\mathrm{N}=452)$} & \multicolumn{2}{|c|}{ Oral risperdone ( $\mathrm{N}=\mathbf{2 5 5 9}$ ) } & \multirow[b]{2}{*}{ chi-square } & \multirow[b]{2}{*}{ df } & \multirow[b]{2}{*}{$\mathbf{P}$} \\
\hline & Number of patients & $\%$ of patients & Number of patients & $\%$ of patients & & & \\
\hline \multicolumn{8}{|c|}{ Ethnicity } \\
\hline Asian & 21 & 4.6 & 101 & 3.9 & 0.88 & 3 & 0.831 \\
\hline Black & 115 & 25.4 & 649 & 25.4 & & & \\
\hline Other & 182 & 40.3 & 1008 & 39.4 & & & \\
\hline White & 134 & 30.0 & 801 & 31.3 & & & \\
\hline \multicolumn{8}{|c|}{ Age Range (years old) } \\
\hline 18-34 & 235 & 52.0 & 1099 & 42.9 & 13.46 & 3 & 0.004 \\
\hline 35-44 & 69 & 15.3 & 501 & 19.6 & & & \\
\hline $45-54$ & 84 & 18.6 & 522 & 20.4 & & & \\
\hline $55-64$ & 64 & 14.2 & 437 & 17.1 & & & \\
\hline
\end{tabular}

TABLE 1: Use of paliperidone palmitate or injectable risperidone versus oral risperidone amongst different ethnicities and ages

\section{Discussion}

This study provides recent estimates of how r-LAIs were prescribed as compared to oral risperidone amongst different ethnicities in a large county inpatient system. Our findings are in disagreement with previous studies [5-7]. Reports have indicated that ethnic minorities are less likely to receive optimal access to atypical LAIs [7]. However, our data shows that there were no significant racial or ethnic differences in how 
these medications were prescribed. Each ethnicity was just as likely to receive r-LAIs as they were to receive oral risperidone. As racial disparity may affect the quality of care, subsequent studies should examine whether racial parity on receiving long-acting injectable antipsychotics improves the quality of care [12]. Particularly, our data indicate that both Asian Americans and African Americans are as likely to receive optimal care, as defined by likelihood, to receive depot antipsychotics in a large county inpatient system.

This study also provides recent estimations of r-LAI use as compared to oral risperidone amongst different age ranges. Historically, LAIs have been reserved for patients with the poorest compliance and for those in later stages of the disease [13]. However, our data report a higher percentage of individuals aged 18-34 years (in earlier stages of the disease) being treated with atypical LAIs over oral Risperidone. Interestingly, recent studies have indicated that LAIs can be effective even during first-episode psychosis [7-9]. For such patients, LAIs were associated with an improved quality of life and a clinically irrelevant incidence of extrapyramidal side effects [7]. The higher percentage of individuals on r-LAIs (52.0\%) over oral risperidone (42.9\%) within this age range (18-34 years of age) may illustrate an emerging trend to address early psychosis with newer, long-acting formulations.

This study has several limitations. First, there is a lack of data regarding other atypical LAIs or oral paliperidone, as these medications were not available on the formulary. Second, there is a lack of generalizability of these results towards other counties; this study only used de-identified data of one county. Third, the study did not account for other factors that contribute to racial disparities due to a lack of individual-level data. Fourth, there is a lack of data regarding psychiatric diagnoses as well as co-morbid substance use disorders in this study population [14-15]. Finally, although Los Angeles County has a large Hispanic population, the LAC DHS dataset does not have a separate racial category for the Latino population. This makes it impossible to calculate a reliable percentage of the Hispanic population for this particular study.

\section{Conclusions}

Our data indicate a lack of ethnic disparities (particularly for African and Asian Americans) in prescribing atypical long-acting injectable antipsychotics and an increased percentage of younger patients being treated with atypical depot antipsychotics over their oral equivalents. As LAIs are both safe and effective for patients with schizophrenia, the results of this study indicate that quality of care can be achieved in a communitybased psychiatric inpatient population. Additional methodologically rigorous studies using standardized outcome measures are needed to examine the relationship between the utilization of LAIs and the quality of care.

\section{Additional Information \\ Disclosures}

Human subjects: Consent was obtained by all participants in this study. Animal subjects: All authors have confirmed that this study did not involve animal subjects or tissue. Conflicts of interest: In compliance with the ICMJE uniform disclosure form, all authors declare the following: Payment/services info: All authors have declared that no financial support was received from any organization for the submitted work. Financial relationships: All authors have declared that they have no financial relationships at present or within the previous three years with any organizations that might have an interest in the submitted work. Other relationships: All authors have declared that there are no other relationships or activities that could appear to have influenced the submitted work.

\section{References}

1. Morris MT, Tarpada SP: Long-acting injectable paliperidone palmitate: a review of efficacy and safety . Psychopharmacol Bull. 2017, 15:42-52.

2. Lacro JP, Dunn LB, Dolder CR, Leckband SG, Jeste DV: Prevalence of and risk factors for medication nonadherence in patients with schizophrenia: a comprehensive review of recent literature. J Clin Psychiatry. 2002, 63:892-909.

3. Lieberman JA, Stroup TS, McEvoy JP, et al.: Effectiveness of antipsychotic drugs in patients with chronic schizophrenia. N Engl J Med. 2005, 22:1209-1223. 10.1056/NEJMoa051688

4. Correll CU, Citrome L, Haddad PM, Lauriello J, Olfson M, Calloway SM, Kane JM: The use of long-acting injectable antipsychotics in schizophrenia: evaluating the evidence. J Clin Psychiatry. 2016, 3:1-24. 10.4088/JCP.15032su1

5. Lawson W, Johnston S, Karson C, et al.: Racial differences in antipsychotic use: claims database analysis of Medicaid-insured patients with schizophrenia. Ann Clin Psychiatry. 2015, 27:242-252.

6. Aggarwal NK, Rosenheck RA, Woods SW, Sernyak MJ: Race and long-acting antipsychotic prescription at a community mental health center: a retrospective chart review. J Clin Psychiatry. 2012, 73:513-517. 10.4088/JCP.11m07161

7. Puyat JH, Daw JR, Cunningham CM, et al.: Racial and ethnic disparities in the use of antipsychotic medication: a systematic review and meta-analysis. Soc Psychiatry Psychiatr Epidemiol. 2013, 48:18611872. 10.1007/s00127-013-0753-4

8. Zhu Y, Krause M, Huhn M, et al.: Antipsychotic drugs for the acute treatment of patients with a first episode of schizophrenia: a systematic review with pairwise and network meta-analyses. Lancet Psychiatry. 2017, 


\section{Cureus}

4:694-705. 10.1016/S2215-0366(17)30270-5

9. Bora E, Yalincetin B, Akdede BB, Alptekin K: Duration of untreated psychosis and neurocognition in firstepisode psychosis: a meta-analysis. Schizophr Res. In Press, 2017, 10.1016/j.schres.2017.06.021

10. Conus P, Cotton S, Schimmelmann BG, McGorry PD, Lambert M: Rates and predictors of 18-months remission in an epidemiological cohort of 661 patients with first-episode psychosis. Soc Psychiatry Psychiatr Epidemiol. 2017, 52:1089-1099. 10.1007/s00127-017-1388-7

11. Malla A, Tibbo P, Chue P, et al.: Long-acting injectable antipsychotics: recommendations for clinicians. Can J Psychiatry. 2013, 58:30-35. 10.1177/088740341305805s05

12. Woo BK, Sultzer DL: A key step for quality care in psychiatric emergency settings . Gen Hosp Psychiatry. 2009, 6:503-504. 10.1016/j.genhosppsych.2009.06.006

13. Kane JM, Garcia-Ribera C: Clinical guideline recommendations for antipsychotic long-acting injections. Br J Psychiatry. 2009, 52:63-67. 10.1192/bjp.195.52.s63

14. Woo BK, Sevilla CC, Obrocea GV: Factors influencing the stability of psychiatric diagnoses in the emergency setting: review of 934 consecutively inpatient admissions. Gen Hosp Psychiatry. 2006, 5:434-436. 10.1016/j.genhosppsych.2006.07.005

15. Woo BK, Chen W: Substance misuse among older patients in psychiatric emergency service . Gen Hosp Psychiatry. 2010, 1:99-101. 10.1016/j.genhosppsych.2009.08.002 